\section{Specimen Preparation for Condoms}

Gan Phay Fang

Science \& Technology Innovative Centre, Ansell Shah Alam Sdn Bhd Selangor, Malaysia

pgan@ap.ansell.com

\section{Introduction}

Specimen preparation techniques for Scanning Electron Microscope ( SEM ) imaging of condoms as reported by Rosenzweig et al [1] revealed a variety of artifacts. The artifacts were classified as ridging [2], cracking and melting. The purpose of

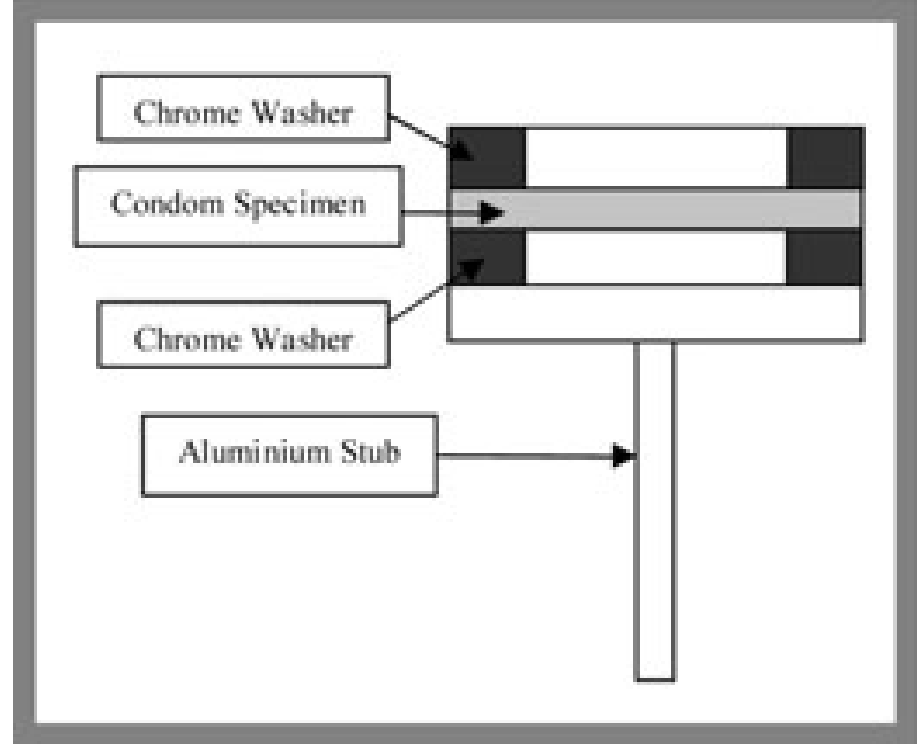

Figure 1 The mounting assembly Figure 3 SEM Center: Imaging of condom from manufacturer $B$ at $500 x$ showing melting of a condom Figure 4 SEM Right: Imaging of condom from manufacturer $B$ at $1500 x$ showing ridging of a condom this article is to introduce a simple specimen prepar nique for condoms to be evaluated via SEM without any surface artifacts. This technique involves the use of two chrome washers to sandwich the condom. The sandwiched condom specimen is then subjected to coating before mounting on an aluminium stub. The execution of this technique requires patience and practice so as not to damage the condom. The method may be applied to any similar polymer material.

\section{Experimental}

Different brands of commercial lubricated condoms were purchased for this study. Initially, a circular test specimen was cut with a $32 \mathrm{~mm}$ circular die from each condom sample. The circular condom specimens were then immersed in isopropanol at room temperature for 10 seconds with gentle rubbing before drying in an oven at $40^{\circ} \mathrm{C}$ for half an hour.

The washed condom specimens were prepared and analyzed as follows: 1) the specimen preparation technique reported in Rosenzweig et al [1] and 2) a new specimen preparation technique described as follows: The washed condom specimen was mounted without stretching on a chrome washer that had a double-sided conductive carbon tape applied to its surface. The other side of the washed specimen was then attached to another chrome washer that also had a double-sided conductive carbon tape on its surface. This assembly was then sputter coated for 2 minutes at $25 \mathrm{~mA}$ with gold-palladium. All coatings from the specimen preparation techniques 1) and 2) were conducted on an Emitech K550X Sputter Coater. The coated assembly was then mounted on an aluminium stub as shown in figure 1. SEM analysis was performed at $15 \mathrm{kV}$ accelerating voltage in a Hitachi S 3000N SEM.
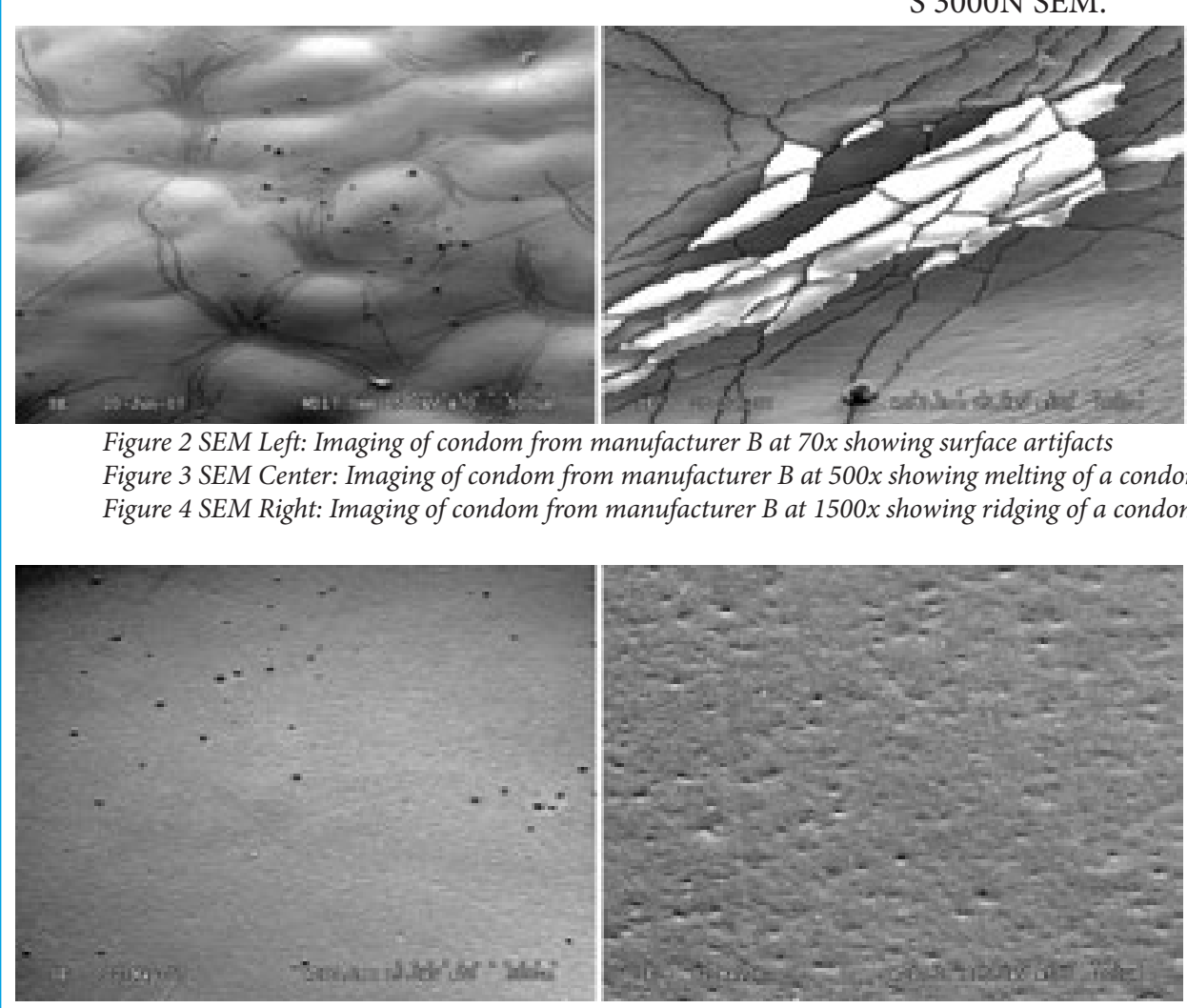

Figure 2 SEM Left: Imaging of condom from manufacturer B at 70x showing surface artifacts

Figure 5 Left: SEM imaging of condom from manufacturer $A$ at $70 x$ showing no surface artifacts Figure 6 Right: SEM imaging of condom from manufacturer $B$ at $500 x$

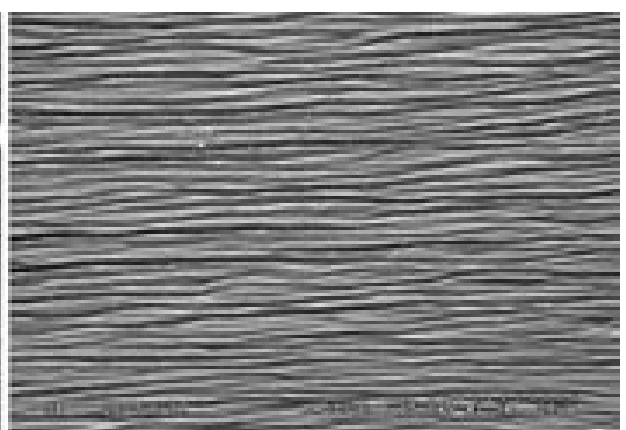

\section{Results}

The condoms from the different manufacturers were viewed at different magnifications of $70 \times, 500 \times, 1000 \times$ and $1500 \times$ at 60 degree tilt. A pore of one of the condom was viewed at $3000 \times$ and $5000 \times$ at 60 degree tilt. Artifacts were observed in the samples prepared using the specimen preparation technique reported by Rosenzweig et al [1] as shown in figures $2,3 \& 4$.

No artifacts were observed on any samples prepared utilizing the new specimen 


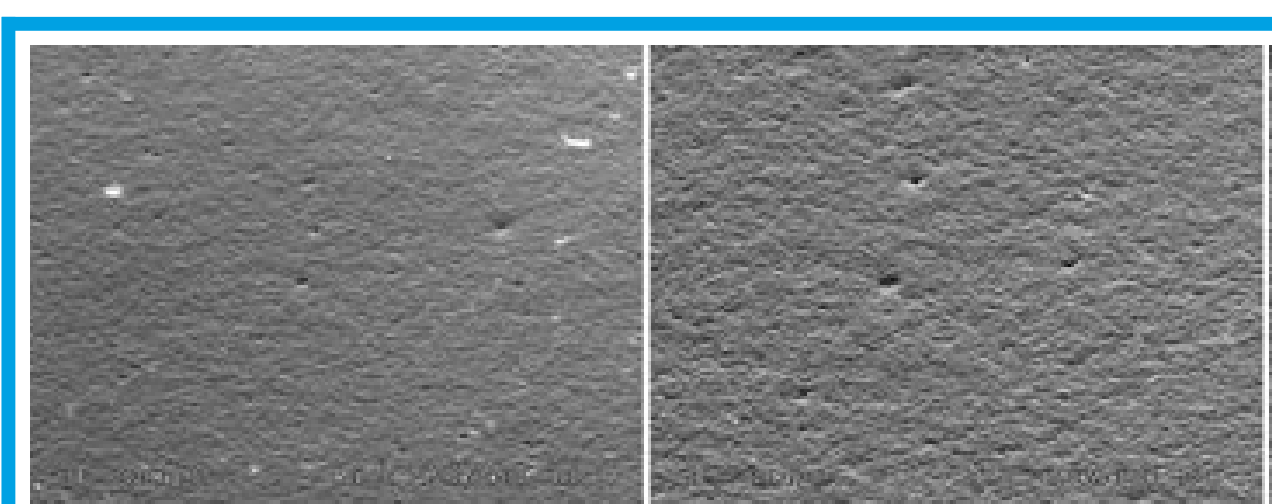

Figure 7 Left: SEM imaging of condom from manufacturer $C$ at $500 x$

Figure 8 Center: SEM imaging of condom from manufacturer $C$ at $1000 x$

Figure 9 Right: SEM imaging of condom from manufacturer $C$ at $3000 x$

preparation technique. Figure 5 shows the surface morphology of the condom from Manufacturer A at a lower magnification $(70 \times)$ with no surface artifacts. Figure 6 (Manufacturer B ). Figures $7 \& 8$ (Manufacturer $C$ ) show the surface morphology of the condoms at medium $(500 \times)$ and high $(1000 \times)$ magnifications. Figure 9 reveals the pores on the condom of manufacturer $\mathrm{C}$ at a magnification of $3000 \times$.

\section{Discussion}

This simple technique has been shown to eliminate the surface artifacts reported in [1], thus making the viewing of ultrathin condom films under SEM possible. The suspension of the condom specimen between the chrome washers has essentially eliminated the artifacts [1] due to the mounting process.
The assessment of condoms under the SEM using this sample preparation technique has indeed helped to advance research and developmental work through an understanding of the surface characteristics of condoms.

\section{Acknowledgment}

The author would like to thank Ngui Chih Lipp, Director of Analytical Services, Science \& Technology Innovative Centre, Ansell Shah Alam Sdn Bhd for his invaluable advice and assistance necessary to complete this work.

\section{References}

1. B. A. Rosenzweig, A. Even and E. Budnick, Contraception, Vol. 53, pp 49 - 53, 1996

2. G.D. Jay, E. Drummond and B. Lane, Contraception, Vol. 45, pp 105 - 10, 1992.

\section{SII $0^{\circ}$}

SII NanoTechnology USA Inc.

Silicon Drift Detector

\section{NO LN2 - Active area $~ 50 \mathrm{~mm}^{2} \bullet<136 \mathrm{eV}$ FWHM at $5.9 \mathrm{keV} \cdot$ ICR $1.5 \mathrm{Mcps} \cdot 0$ CR up to $600 \mathrm{kcps}$}

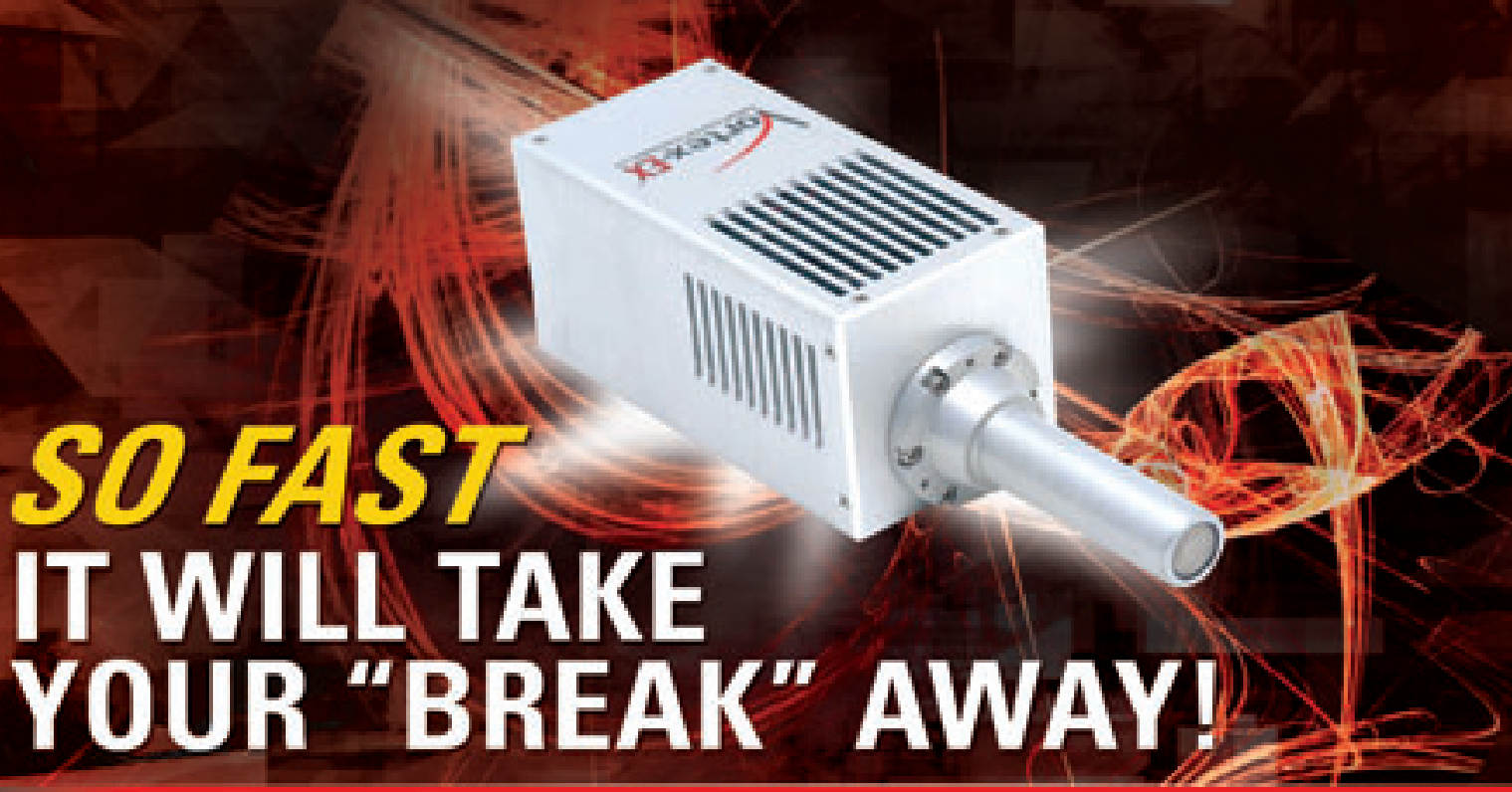

